\title{
Construction of a simple evaluation system for the intestinal absorption of an orally administered medicine using Bombyx mori larvae
}

\author{
Fumika Ichino', Hidemasa Bono ${ }^{2}$, Takeru Nakazato ${ }^{2}$, Atsushi Toyoda ${ }^{3}$, Asao Fujiyama ${ }^{3}$, \\ Kikuo Iwabuchi ${ }^{1}$, Ryoichi Sato ${ }^{4}$, Hiroko Tabunoki ${ }^{1, *}$ \\ ${ }^{1}$ Department of Science of Biological Production, Graduate School of Agriculture, Tokyo University of Agriculture and \\ Technology, Tokyo, Japan; \\ ${ }^{2}$ Database Center for Life Science (DBCLS), Joint Support-Center for Data Science Research, Research Organization of \\ Information and Systems (ROIS), Mishima, Shizuoka, Japan; \\ ${ }^{3}$ Center for Information Biology, National Institute of Genetics, Mishima, Shizuoka, Japan; \\ ${ }^{4}$ Graduate School of Bio-Applications and Systems Engineering (BASE), Tokyo University of Agriculture and Technology, Tokyo, Japan.
}

\begin{abstract}
Summary Human intestinal absorption is estimated using a human colon carcinoma cell line (Caco-2) cells from human colorectal adenocarcinoma, intestinal perfusion, or a mammalian model. These current evaluation systems are limited in their ability to estimate human intestinal absorption. In addition, in vivo evaluation systems using laboratory animals such as mice and rats entail animal ethics problems, and it is difficult to screen compounds on a large scale at the drug discovery stage. Thus, we propose the use of Bombyx mori larvae for evaluation of intestinal absorption of compounds as an alternative system in this study. First, to compare the characteristics among Caco-2 cells, human intestine, and $B$. mori larval midgut, we analyzed their RNA-seq data, and we found 26 drug transporters common to humans and $B$. mori. Next, we quantitatively developed an oral administration technique in $B$. mori and established a method using silkworm $B$. mori larvae that can easily estimate the intestinal permeability of compounds. Consequently, we could determine the dose and technique for oral administration in $B$. mori larvae. We also developed a $B$. mori model to evaluate the intestinal permeability of orally administered. Our constructed evaluation system will be useful for evaluating intestinal permeability in medical drug development.
\end{abstract}

Keywords: Intestinal permeability, transporters, next-generation sequencing, B. mori

\section{Introduction}

Although drug discovery can be genome-based, many candidate compounds are rejected in clinical trials for pharmaceutical products. A major reason for such as rejection is poor pharmacokinetics; in particular, poor intestinal absorption of candidate compounds. As a screening method using a human colon carcinoma cell line (Caco-2) for predicting the intestinal absorption

\footnotetext{
*Address correspondence to:

Dr. Hiroko Tabunoki, Department of Science of Biological Production, Graduate School of Agriculture, Tokyo University of Agriculture and Technology, 3-5-8 Saiwai-cho, Fuchu, Tokyo 183-8509, Japan.

E-mail: h_tabuno@cc.tuat.ac.jp
}

of a compound, the Caco-2 cell culture system is derived from high-throughput screening and in situ perfusion systems (Figure 1) (1-3). However, these current evaluation systems cannot be used to evaluate the intestinal absorption of any compound, maintaining the villus structure and function of the small intestinal epithelium (4). In addition, in vivo evaluation systems have problems related to time, expense, and the ethics of using laboratory animals, such as mice and rats. For these reasons, it is difficult to screen compounds on a large scale. Therefore, we need to construct a new evaluation method for the intestinal absorption of candidate compounds for large scale screening.

The silkworm Bombyx mori is a lepidopteran insect that is used as a model organism in agricultural research. Their genome sequence is almost completely 
A

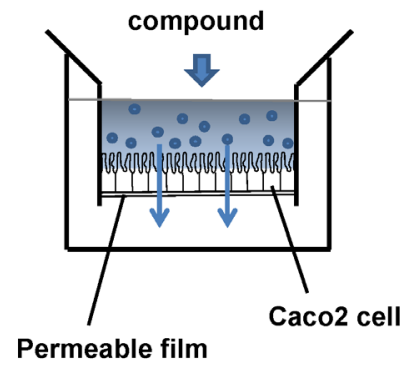

C
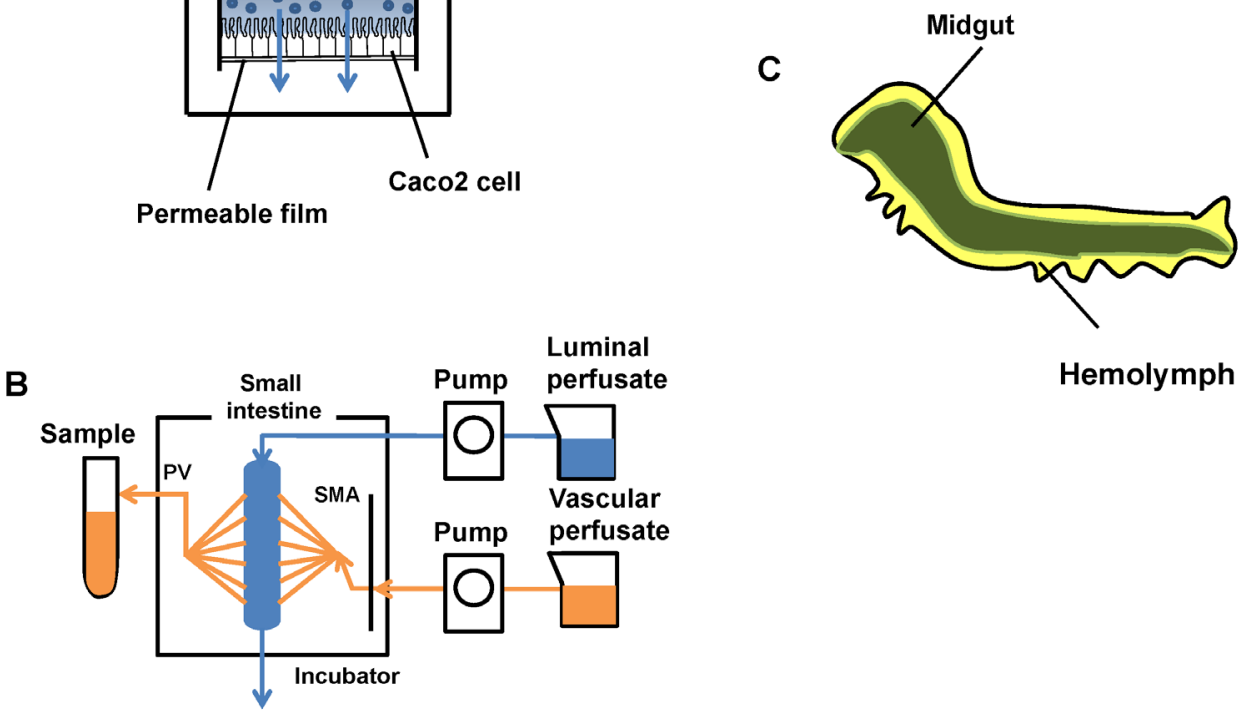

Hemolymph

Figure 1. Evaluation of model systems for intestinal permeability and the internal structure of $B$. mori larva. (A) Caco2 cell system. The cells are cultured on a permeable film, and the objective compound is dissolved into the culture medium. The compound passes through the permeable film and into a lower culture dish. (B) Intestinal perfusion system. A modification by Ishihara et al. (2001) (2). The small intestine was dissected from mice and set into an incubator filled with buffer $\left(37^{\circ} \mathrm{C}\right)$. A solvent containing the objective compound was perfused by a pump into the small intestine. SMA: superior mesenteric artery; PV: portal vein. (C) Internal structure of B. mori larva. Most of the body is occupied by the midgut (green), and its surroundings are filled with hemolymph (yellow).

characterized, various spontaneous genetic mutants are available, and the silkworm is amenable to transgenics, genome editing, and transcriptome analysis (5-10). Moreover, the larval body size is bigger than that of Drosophila melanogaster: their gastrointestinal tract occupies $\geq 80 \%$ of the whole larval body and is structurally similar to the human small intestine (11); however, B. mori has never been used as an evaluation model for the absorption of a compound. Hamamoto et al. (12) investigated the pharmacokinetics (absorption, distribution, metabolism, excretion) of some drugs by using silkworm larvae and showed that most of the data were consistent with those of the mouse model $(13,14)$. Drugs are metabolized by P450 enzymes and are then processed in a conjugation reaction. These metabolic processes and excretion of compounds are similar to those of mammalian models (12); for example, the effective dose of antibiotics in the bacterial infection model using silkworm larvae was consistent with that in the mammalian model (15). These results indicate the possibility that silkworms can be used as a substitute for mammals.

In addition, current intestinal permeability systems cannot adequately predict the intestinal absorption of compounds at the pharmaceutical investigation stage. To evaluate pharmacokinetics without laboratory animals at the preliminary developmental stage, an evaluation system that can easily predict absorption from the small intestine of an orally administered compound is required. Such a system not only has economic advantages, such as reduced time and cost, but also improved animal ethics at the preliminary developmental stage; however, there is still no suitable screening method available. We therefore propose the use of $B$. mori larvae for the evaluation of intestinal permeability. This model can easily and conveniently predict the intestinal absorption of compounds; thus, we quantitatively developed oral administration techniques in $B$. mori and established a method using $B$. mori larvae that can rapidly measure the intestinal permeability of compounds.

\section{Materials and Methods}

\subsection{Insects}

The B. mori larvae used in the study were supplied by Ueda-Sha Co. Ltd. (Ueda-shi, Nagano, Japan). Silkworm larvae were reared on an artificial diet of Silkmate $2 \mathrm{~S}$ (Nosan, Tsukuba-shi, Ibaraki, Japan). Insects were maintained at $25^{\circ} \mathrm{C}$ with a 12 -h light/dark cycle. Day 3 fifth-instar B. mori larvae were used for the evaluation of intestinal absorption. The B. mori strain o751 (wild-type) used in the RNA-seq analysis was obtained from the Institute of Genetic Resources, Faculty of Agriculture, Kyushu University (NBRP silkworm database: http:// silkworm.nbrp.jp/index_en.html). 


\subsection{RNA-seq analysis}

Total RNA was isolated from the midgut of day 3 fifth-instar B. mori larvae of B. mori 0751 wild-type by using a PureLink ${ }^{\circledR}$ RNA extraction kit (Thermo Fisher Scientific Inc., Valencia, CA, USA) according to the manufacturer's protocol. The RNA quality was assessed by using an Agilent Bio-analyzer 2100 (Agilent Technologies, Santa Clara, CA, USA). Paired-end sequencing cDNA libraries were constructed with 4 $\mu \mathrm{g}$ of total RNA from o751 wild-type samples $(n=3)$ by using a TruSeq RNA Sample Preparation Kit Set A (Illumina Inc., San Diego, CA, USA) according to the manufacturer's protocol. RNA-seq was performed by using a HiSeq 2500 system (Illumina Inc.). The data quality of the fastq files was verified by using the FastQC tool (Babraham Bioinformatics, http:// www.bioinformatics.babraham.ac.uk/projects/fastqc/). The around $43-\mathrm{M}$ paired-end reads $(2 \times 150 \mathrm{bp})$ were mapped to the reference $B$. mori genome available from the Ensembl Genome database (16) using the Bowtie2 program version 2.3.2 with default parameters (17). RNA-Seq by Expectation-Maximization software version 1.3.0 was used for the calculation of expression values in transcripts per million (TPM) (18).

The gene expression data for Caco-2 cells and human small intestine was obtained from the Reference Expression Dataset (19). Data used for the comparative analysis were "Processed expression data of all samples for CAGE human PRJDB3010 (FANTOM5)" (doi: $10.6084 / \mathrm{m} 9$.figshare.4028613.v4) downloaded from https://doi.org/10.6084/m9.figshare.4028613.v4.

\subsection{Identification of B. mori human transporter homologs by systematic BLAST search}

To identify the human transporter homolog in the silkworm, genes that were homologous to human genes were identified by performing a systematic basic local alignment search tool (BLAST) search (tblastx) with a cutoff E-value of significant homology at $10^{-10}$ (query: human transporter cDNA sequence; database: whole silkworm cDNA sequence set from the Ensembl database).

\subsection{Determination of dose for oral administration to $B$. mori larvae}

To determine the dose for oral administration, we injected several amounts of water into day 3 fifthinstar B. mori larvae via their mouthparts by using a disposable syringe (Terumo, Shibuya-ku, Tokyo, Japan) with a modified TSK 30G needle (Tochigi-seiko Co. Ltd., Utsunomiya-shi, Tochigi, Japan) as an oral probe. The tip of the $30 \mathrm{G}$ needle was filed by using sand paper, and the edge of the needle tip was removed for oral administration. The silkworm larvae were not fed for $24 \mathrm{~h}$ before the administration of the compounds. The body weights of day 3 fifth-instar $B$. mori larvae were $2.88 \pm 0.16 \mathrm{~g}$ (mean $\pm \mathrm{SD}$ ). In this experiment, we defined "success" as survival of the larvae the day following the oral administration (for $24 \mathrm{~h}$ ), and there were no problems in the oral injection into the larva; whereas "failure" was defined as death or other problems occurring in the course of the oral injection into the larva. We statistically calculated a correlation coefficient for the relationship between dose and larval body weight by using Microsoft Excel. In this experiment, we denoted "success" as 1 and "failure" as 0.

2.5. Determination of the $L D_{50}$ of theophylline, tetracycline hydrochloride, and chloramphenicol in day 3 fifth-instar B. mori larvae

Theophylline, tetracycline hydrochloride, and chloramphenicol were purchased from Wako Pure Chemical Industries, Ltd. (Chuo-ku, Tokyo, Japan). To determine the $50 \%$ of lethal dose $\left(\mathrm{LD}_{50}\right)$ in day 3 fifthinstar B. mori larvae, we orally injected theophylline, tetracycline hydrochloride, and chloramphenicol by using the modified oral probe mentioned above. Theophylline was dissolved in $20 \%$ dimethyl sulfoxide (Wako Pure Chemical Industries, Co., Ltd.) at 0 (control), 1, 1.25, 1.50, 1.75, and $2.00 \mathrm{mg} / \mathrm{g}$ and injected at a volume of $0.18 \mathrm{~mL} / \mathrm{g}$ body weight. Tetracycline hydrochloride was dissolved in distilled water (Otsuka Pharmaceutical Co., Ltd., Naruto-shi, Tokushima, Japan) at 0 (control), $1,1.25,1.50,1.75,2.00,2.25$, and $2.50 \mathrm{mg} / \mathrm{g}$ and injected at a volume of $0.18 \mathrm{~mL} / \mathrm{g}$ body weight. Chloramphenicol was dissolved in $100 \%$ ethanol (Wako Pure Chemical Industries, Co., Ltd.) at 0 (control), 0.2, 0.4, 0.6, 0.7, 0.8, and $1.0 \mathrm{mg} / \mathrm{g}$ and injected at a volume of $0.18 \mathrm{~mL} / \mathrm{g}$ body weight. Each of the control groups was injected with their respective undiluted solvent.

The number of dead silkworms after $24 \mathrm{~h}$ was counted and the mortality rate was calculated using the formula mortality rate $(\%)=(X / Y) \times 100$, where $X$ $=$ dead larvae in the group and $\mathrm{Y}=$ total larvae in the group. To calculate the LD50, larval mortality rates were analyzed by using the Probit Analysis (20) option of the JMP 10.0 software package (SAS Institute Japan Ltd., Minato-ku, Tokyo, Japan). After obtaining these results, we determined the $\mathrm{LD}_{50}$ values of theophylline, tetracycline hydrochloride, and chloramphenicol for administration to $B$. mori larvae.

Theophylline, tetracycline hydrochloride, and chloramphenicol, prepared at $1.25,1.5$, and $0.6 \mathrm{mg} / \mathrm{g}$ in each solvent, respectively, were injected into 10 larvae at a volume of $0.18 \mathrm{~mL} / \mathrm{g}$ body weight. After $0.5,1,1.5$, 2, 3, 4.5, 6, and $9 \mathrm{~h}$, intact hemolymph was collected from each compound-treated larva without a reducing agent and immediately frozen at $-80^{\circ} \mathrm{C}$ until use. 


\subsection{Sample preparation for evaluation of intestinal permeability}

To remove protein components, one volume of methanol (Wako Pure Chemical Industries, Co., Ltd.) was added to the collected hemolymph. This was mixed well by using a vortex mixer and kept on ice for $15 \mathrm{~min}$ and then centrifuged at $1,000 \mathrm{~g}$ for $15 \mathrm{~min}$ at $4^{\circ} \mathrm{C}$. The supernatant was transferred to a new tube and filtered by using a 0.45- $\mu \mathrm{m}$ filter (ADVANTECH Co., Ltd., Bunkyo-ku, Tokyo, Japan). Finally, the adjusted sample was added to seven volumes of methanol for the analysis of intestinal permeability by high-performance liquid chromatography (HPLC).

\subsection{Determination of HPLC detection conditions}

The following equipment was used for the detection of intestinal permeability by HPLC analysis: an intelligent pump (JascoPU.2080Plus; JASCO Co., Ltd. Hachiojishi, Tokyo, Japan), ultraviolet detector (UV.2077Plus, Mx.2080.31, DG, 2080.53; JASCO Co., Ltd.), and an octa decyl silyl (ODS) 18 column $(25 \mathrm{~cm} \times 4.6 \mathrm{~mm}$ I.D.; GL Science, Shinjyuku-ku, Tokyo, Japan), and a step-wise gradient mode (flow rate; $0.7 \mathrm{~mL} / \mathrm{min}$ ). The mobile phase used the following conditions: $5 \%$ $\mathrm{MeOH}$ containing $0.2 \%$ acetic acid, $100 \%$ Methanol, $100: 0 \rightarrow 60: 40$ for $0-15 \mathrm{~min}$, and $60: 40 \rightarrow 60: 40$ for 15-30 min. All analyses were performed at room temperature $\left(25^{\circ} \mathrm{C}\right)$. Chromato-PRO software (Run Time Co., Ltd., Hachioji-shi, Tokyo, Japan) was used in data processing. The elution was monitored at $273 \mathrm{~nm}$ (theophylline) or $275 \mathrm{~nm}$ (tetracycline hydrochloride and chloramphenicol).

\section{Results}

3.1. Identification of common drug transporters in the human intestine and B. mori larval midgut

To compare the characteristics among Caco- 2 cells, the human small intestine, and the B. mori larval midgut, we analyzed the $B$. mori larval midgut RNA-seq data to identify the homologs of the human drug transporters expressed in the small intestine. As a result, we found 26 drug transporter homologs that were common in the B. mori larval midgut and human intestine. In contrast, the ATP-Binding Cassette (ABC) Subfamily A Member 13 ( $A B C A 13)$ and $A B C B 4$ were expressed only in the human intestine. Moreover, Solute Carrier Family (SLC) 22 Member 15 (SLC22A15), ABCA5, ABCB1, ABCG2, and Solute Carrier Organic (SLCO) Anion Transporter Family Member 4 A1 (SLCO4A1) were not expressed in the $B$. mori larval midgut. $A B C C 8, A B C G 1, A B C G 4$, $A B C G 5, A B C G 8, S L C O 3 A 1, S L C O 5 A 1$, and SV2Related Protein $(S V O P)$ were not expressed in the Caco-2 cells.
Table 1. Human transporter homologs expressed in the $B$. mori larval midgut

\begin{tabular}{|c|c|c|c|}
\hline Gene symbol & $\begin{array}{c}\text { Caco-2 } \\
\text { (CAGE value) }\end{array}$ & $\begin{array}{c}\text { Human } \\
\text { (CAGE value) }\end{array}$ & $\begin{array}{c}\text { B. mori } \\
\text { (TPM value) }\end{array}$ \\
\hline$A B C A 3$ & 2.4 & 2.2 & 81.5 \\
\hline$A B C A 5$ & 0.8 & 4.2 & 0 \\
\hline$A B C A 13$ & 0 & 0.2 & 0 \\
\hline$A B C B 1$ & 2.7 & 5.3 & 0 \\
\hline$A B C B 4$ & 0 & 0.8 & 0 \\
\hline$A B C B 5$ & 0 & 0.5 & 13.6 \\
\hline$A B C B 6$ & 2.5 & 1.8 & 8.8 \\
\hline$A B C B 7$ & 3.1 & 3.2 & 1.4 \\
\hline$A B C B 8$ & 2.6 & 2.8 & 1.7 \\
\hline$A B C B 10$ & 2.9 & 3.2 & 2.9 \\
\hline$A B C C 1$ & 2.4 & 2.2 & 0.1 \\
\hline$A B C C 3$ & 3.2 & 4.6 & 0.2 \\
\hline$A B C C 4$ & 3.7 & 2.5 & 40.3 \\
\hline$A B C C 8$ & 0 & 0.8 & 0.4 \\
\hline$A B C C 10$ & 2.4 & 2.6 & 0.2 \\
\hline$A B C D 3$ & 3.6 & 3.6 & 0.5 \\
\hline$A B C G 1$ & 0 & 3.7 & 2.3 \\
\hline$A B C G 2$ & 2.8 & 5.2 & 0 \\
\hline$A B C G 4$ & 0 & 0.2 & 1.2 \\
\hline$A B C G 5$ & 0 & 4.5 & 0.8 \\
\hline$A B C G 8$ & 0 & 4.7 & 0.1 \\
\hline$S L C 2 A 8$ & 4.2 & 3.2 & 2.4 \\
\hline SLC15A1 & 2.1 & 5.9 & 0.4 \\
\hline SLC15A2 & 0.5 & 0.1 & 17.4 \\
\hline$S L C 22 A 1$ & 0 & 0 & 0.2 \\
\hline$S L C 22 A 3$ & 0.2 & 1.5 & 6.2 \\
\hline$S L C 22 A 4$ & 0.5 & 2.7 & 9.2 \\
\hline$S L C 22 A 5$ & 2.2 & 3.8 & 1.3 \\
\hline$S L C 22 A 6$ & 0 & 0 & 220.8 \\
\hline SLC22A12 & 0 & 0 & 6.0 \\
\hline$S L C 22 A 13$ & 0 & 0.1 & 6.8 \\
\hline$S L C 22 A 15$ & 0.1 & 0.8 & 0 \\
\hline$S L C 22 A 16$ & 0 & 0 & 0.2 \\
\hline$S L C O 3 A 1$ & 0 & 1.7 & 0.1 \\
\hline SLCO4A1 & 1.6 & 2.1 & 0 \\
\hline SLCO5A1 & 0 & 0.2 & 0.8 \\
\hline SVOP & 0 & 0.3 & 0.2 \\
\hline
\end{tabular}

Additionally, SLC22A1, SLC22A6, SLC22A12, $S L C 22 A 13$, and $S L C 22 A 16$ were expressed only in the $B$. mori larval midgut (Table 1). Therefore, there were few differences in the expressed genes among B. mori, Caco2 , and human small intestine.

The RNA-seq reads supporting the conclusions of this article are available in the Sequence Read Archive with the following accession numbers: DRR095108, DRR095109, and DRR095110.

3.2. Evaluation of the $L D_{50}$ values of theophylline, tetracycline hydrochloride, and chloramphenicol

To determine the dose for oral administration, we injected various amounts of water into day 3 fifth-instar B. mori larvae by oral administration. In this experiment, we defined success as survival of the larvae surviving until the following day and failure as death or a problem occurring in the oral injection to the larvae. We then, calculated the relationship between the amount of water and larval body weight (Figure 2, Table 2), selecting 0.18 
$\mathrm{mL} / \mathrm{g}$ as injection dose. All oral administration doses were set at $0.18 \mathrm{~mL} / \mathrm{g}$ in this study.

Next, we evaluated the LD50 of theophylline, tetracycline hydrochloride, and chloramphenicol,

A
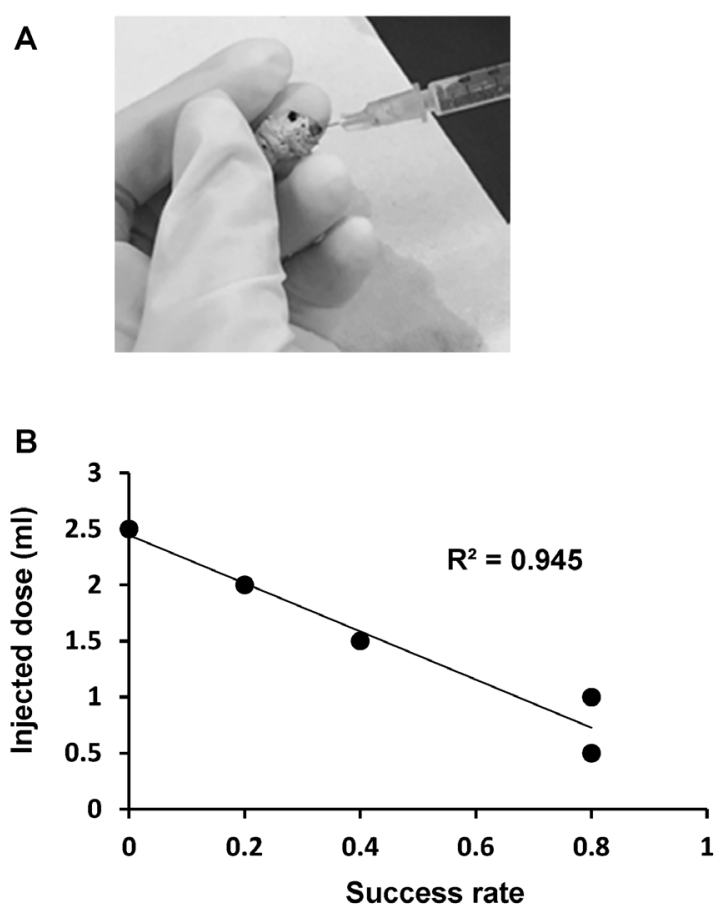

Figure 2. Relationship between success rate and injection dose. (A) Oral administration to B. mori larvae. (B) Relationship between success rate and injection dose of oral administration in $B$. mori larvae. R2 indicates the correlation coefficient.

A

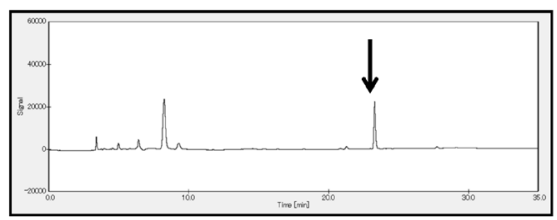

B

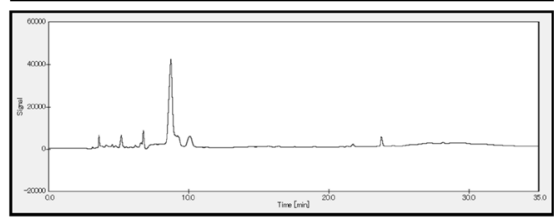

C

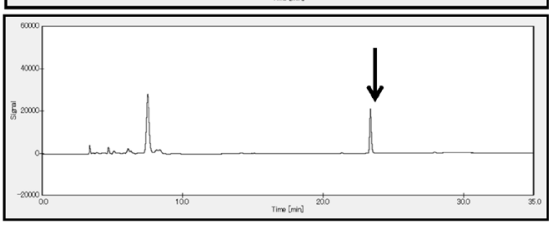

D

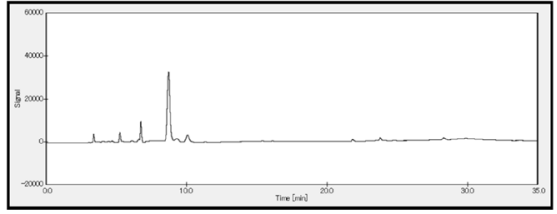

which are used as predictors of intestinal absorption in mammalian models and humans. Thus, we determined the LD50 in day 3 fifth-instar B. mori larvae to be $1.49 \mathrm{mg} / \mathrm{g}$ (95\% confidence interval; CI, 1.37-1.60) for theophylline, $1.95 \mathrm{mg} / \mathrm{g}(95 \% \mathrm{CI}, 1.83-2.05)$ for tetracycline hydrochloride, and $0.74 \mathrm{mg} / \mathrm{g}(95 \% \mathrm{CI}, 0.69$ 0.80) for chloramphenicol.

3.3. Construction of the B. mori model to evaluate the intestinal permeability of compounds by oral administration

To easily evaluate the intestinal permeability of theophylline, tetracycline hydrochloride, and chloramphenicol, we assessed their intestinal permeability by using $B$. mori larvae. First, we investigated the conditions for HPLC analysis by using each compound, and the flow rate and composition of the solvent were determined. Retention times were as follows: approximately $23 \mathrm{~min}$ for theophylline, approximately $25 \mathrm{~min}$ for tetracycline hydrochloride, and 35 min for chloramphenicol. Subsequently, theophylline, tetracycline hydrochloride, and chloramphenicol were

Table 2. Relationship between success rate and injection dose

\begin{tabular}{lcc}
\hline $\begin{array}{c}\text { Success rate } \\
(\%)\end{array}$ & $\begin{array}{c}\text { Injection dose }(\mathrm{mL}) \\
\text { per individual }\end{array}$ & $\begin{array}{c}\text { Injection dose }(\mathrm{mL}) \\
\text { per body weight }\end{array}$ \\
\hline 80 & 0.73 & 0.25 \\
90 & 0.51 & 0.18 \\
95 & 0.40 & 0.14 \\
\hline
\end{tabular}

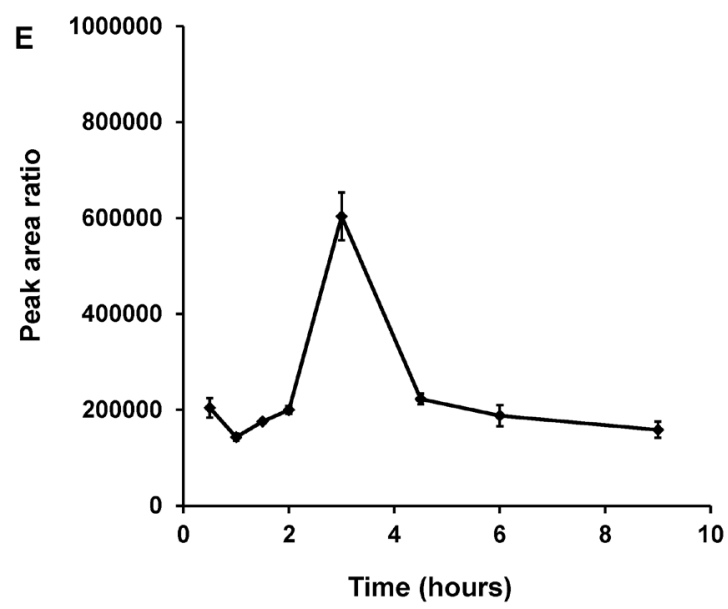

Figure 3. Determination of the intestinal permeability of theophylline by HPLC analysis. (A) Oral administration of theophylline after $30 \mathrm{~min}$. (B) Oral administration of the solvent after $30 \mathrm{~min}$. (C) Oral administration of theophylline after $1.5 \mathrm{~h}$. (D) Oral administration of the solvent after $1.5 \mathrm{~h}$. (E) Time course of the detection of intestinal permeability of theophylline by HPLC analysis. The peak area ratio was calculated by using Chromato-pro software. 
A

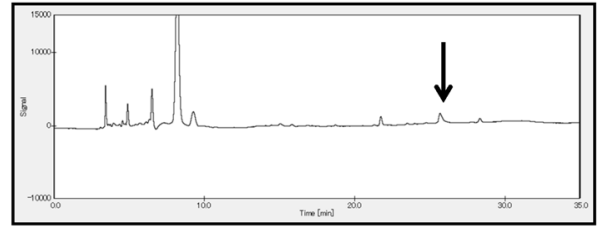

B

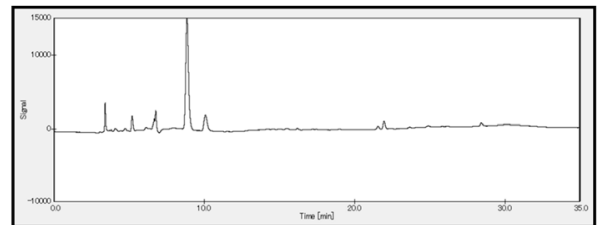

C

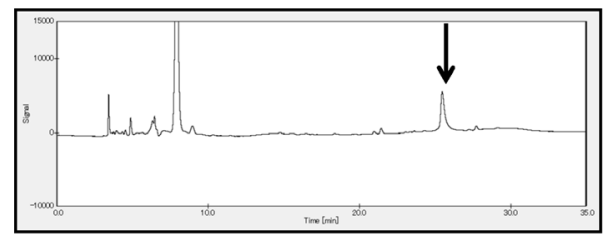

D

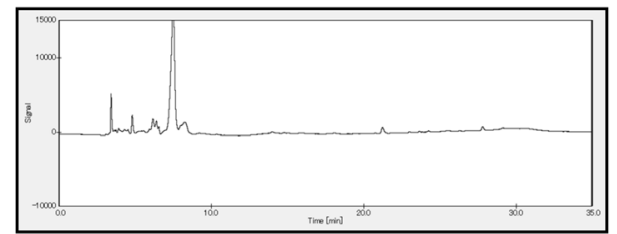

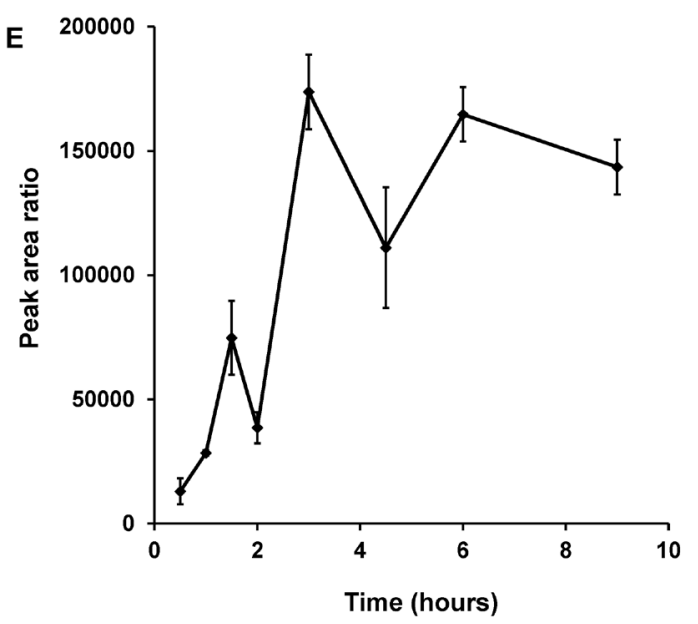

Figure 4. Detection of the intestinal permeability of tetracycline hydrochloride by HPLC analysis. (A) Oral administration of tetracycline hydrochloride after $30 \mathrm{~min}$. (B) Oral administration of the solvent after $30 \mathrm{~min}$. (C) Oral administration of tetracycline hydrochloride after $1.5 \mathrm{~h}$. (D) Oral administration of the solvent after $1.5 \mathrm{~h}$. (E) Time course of the detection of intestinal permeability of tetracycline hydrochloride by HPLC analysis. The peak area ratio was calculated by using Chromato-pro software.

A

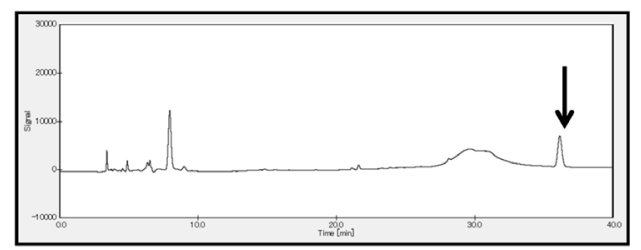

B

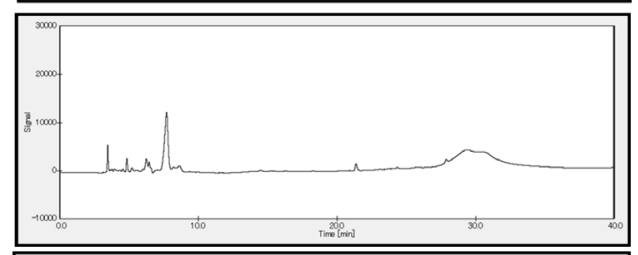

C

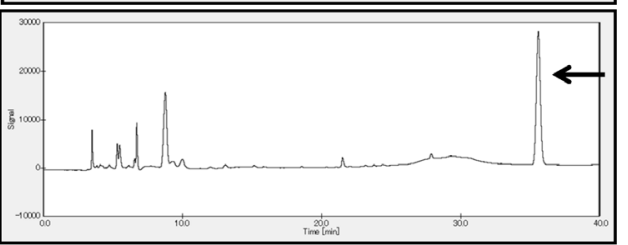

D

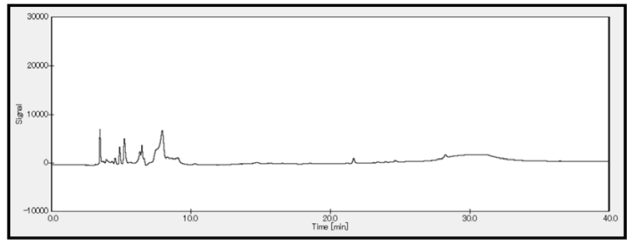

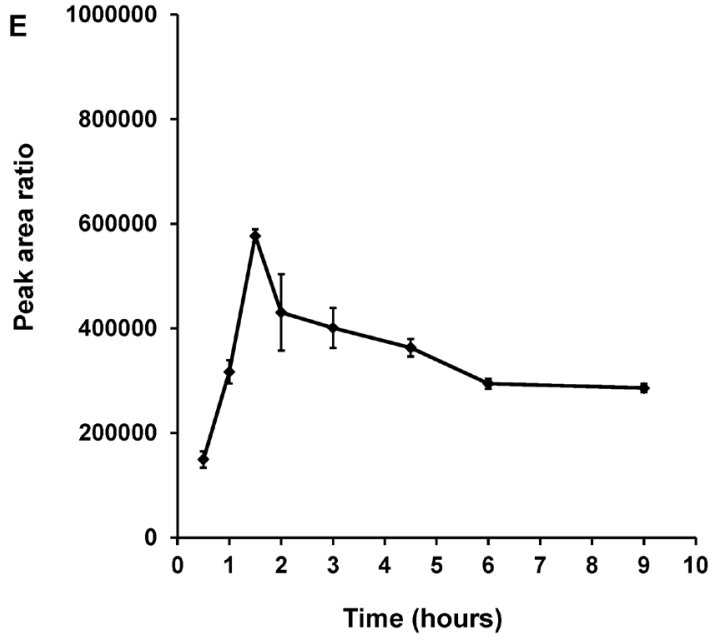

Figure 5. Detection of the intestinal permeability of chloramphenicol by HPLC analysis. (A) Oral administration of chloramphenicol after 30 min. (B) Oral administration of the solvent after $30 \mathrm{~min}$. (C) Oral administration of chloramphenicol after 1.5 h. (D) Oral administration of the solvent after $1.5 \mathrm{~h}$. (E) Time course of the detection of intestinal permeability of chloramphenicol by HPLC analysis. The peak area ratio was calculated by using Chromato-pro software. 
injected orally into the $B$. mori larvae. Theophylline was injected at $1.25 \mathrm{mg} / \mathrm{g}$ body weight of $B$. mori at a volume of $0.18 \mathrm{~mL}$, and the hemolymph was collected after 30 min. We detected the peak that indicates theophylline by HPLC (Figures 3A and 3C, indicated by arrow). Furthermore, the peak confirmed that theophylline was absorbed from the midgut $30 \mathrm{~min}$ after administration. The peak reached its maximum intensity at $3 \mathrm{~h}$, and then gradually decreased (Figure 3E).

Similarly, tetracycline hydrochloride was injected at $1.5 \mathrm{mg} / \mathrm{g}$ body weight of $B$. mori at a volume of $0.18 \mathrm{~mL}$, and the peak indicating that the tetracycline hydrochloride penetrated the hemolymph from the midgut was detected from $30 \mathrm{~min}$ to $9 \mathrm{~h}$ after administration (Figures $4 \mathrm{~A}$ and $4 \mathrm{C}$, indicated by arrow). The peak reached its maximum intensity at $3 \mathrm{~h}$ and then decreased (Figure 4E).

Chloramphenicol was injected at $0.6 \mathrm{mg} / \mathrm{g}$ body weight of $B$. mori at a volume of $0.18 \mathrm{~mL}$, and the peak indicating that the chloramphenicol penetrated the hemolymph from the midgut was detected from $30 \mathrm{~min}$ to $9 \mathrm{~h}$ after administration (Figures 5A and 5C, indicated by arrow). Interestingly, chloramphenicol had the largest peak area in the collected hemolymph at $1.5 \mathrm{~h}$ after administration (Figure $5 \mathrm{C}$ ). When the amount of chloramphenicol absorbed from the midgut was examined, the maximum concentration of the drug occurred at $1.5 \mathrm{~h}$ after the administration and then gradually decreased (Figure 5E).

Retention times were $23.5 \pm 0.17$ min (mean \pm $\mathrm{SD})$ for theophylline, $25.7 \pm 0.02 \mathrm{~min}$ for tetracycline hydrochloride, and $36.4 \pm 0.42 \mathrm{~min}$ for chloramphenicol. Thus, theophylline, tetracycline hydrochloride, and chloramphenicol permeated through the midgut to the hemolymph 30 min after administration (Figures 3A, $4 \mathrm{~A}$, and $5 \mathrm{~A}$ ). No remarkable peak was detected in each control solvent (Figures 3B, 3D, 4B, 4D, 5B, and 5D). Hence, the intestinal permeabilities of theophylline, tetracycline hydrochloride, and chloramphenicol could be evaluated by using the $B$. mori model.

\section{Discussion}

First, we analyzed the RNA-seq data of the day 3 fifth-instar B. mori larval midgut to compare the characteristics between the human small intestine and $B$. mori larval midgut. Interestingly, $16 \mathrm{ABC}$ transporters and 7 SLC transporter genes were commonly expressed in the $B$. mori larval midgut and human intestine. The ABCG transporter genes were well-conserved between B. mori and human; however, Caco-2 cells did not express ABCG transporters. Although 11 SLC transporter genes were identified in the B. mori larval midgut, SLC transporter genes and their function have not been analyzed in $B$. mori.

Fifty-one ABC transporter genes have been identified in B. mori and are categorized into eight classes (21). However, the function of most ABC transporter genes has not been analyzed without the B. mori $\mathrm{ABC}$ transporter subfamily $\mathrm{C}$ member 2 (BmABCC2), which relays transported Cry toxins from Bacillus thuringiensis (22). Aditionally, a single amino acid mutation of $B m A B C C 2$ causes a resistance to Cry toxin in $B$. mori (23). BmABCC2 has high homology with the human $A B C C 4$, also known as multi-drug resistance protein 4 , which is involved in organic anion transport and drug resistance. The midgut RNAseq data showed that BmABCC2 mRNA expression was greater than that of the other transporters in day 3 fifth-instar B. mori larvae; also, human $A B C C 4$ was expressed in the small intestine in the public RNAseq data that we employed. Thus, human $A B C C 4$ and $B m A B C C 2$ might share with similar function.

While the dietary amount increases from day 3 to day 5 in fifth-instar B. mori larvae in B. mori, their body size is also increases from day 3 to day 5 (24). Thus, in this study, day 3 fifth-instar B. mori larvae would be appropriate for evaluation of the intestinal permeability of compounds. It is suggested that the functions of the $B$. mori larval midgut and the human small intestine are similar in terms of intestinal permeability of compounds.

Next, we developed an oral administration method for day 3 fifth-instar B. mori larvae without damaging the oral cavity and gastrointestinal tract. In addition, we used a relatively large $B$. mori strain, Kinshu $\times$ Showa, which remained fasting for $24 \mathrm{~h}$, so we could easily inject via oral administration. We could thus determine the dose per weight.

Then, we used B. mori larvae to examine the intestinal absorption of three compounds with clear intestinal absorption in humans. Theophylline, tetracycline hydrochloride, and chloramphenicol were examined for their intestinal permeability ( $h t t p: / / w w w$. genome.jp/kegg/drug/) because these compounds showed clear dose-response curves in B. mori larva. In humans, the time of maximum concentration $\left(\mathrm{T}_{\max }\right)$ of theophylline has been reported as $24 \mathrm{~h}$, whereas those of tetracycline hydrochloride and chloramphenicol have been reported as 2 and $3 \mathrm{~h}$, respectively (http:// www.genome.jp/kegg/drug/). In this study, the $\mathrm{T}_{\max }$ values of theophylline, tetracycline hydrochloride, and chloramphenicol for B. mori correlated with the human $\mathrm{T}_{\max }$ values.

The $\mathrm{LD}_{50}$ values in $B$. mori for tetracycline and chloramphenicol were comparable to those in mice. According to the material safety data sheet (MSDS), the $\mathrm{LD}_{50}$ is $0.24 \mathrm{mg} / \mathrm{g}$ for theophylline, $2.8 \mathrm{mg} /$ $\mathrm{g}$ for tetracycline hydrochloride, and $1.5 \mathrm{mg} / \mathrm{g}$ for chloramphenicol with oral administration in mice. However, the $\mathrm{LD}_{50}$ for theophylline did not agreed with that in mice data in this study. Thus, $\mathrm{LD}_{50}$ does not necessarily need to match with mice data in the transition of the plasma concentration of theophylline 
because the transition of the plasma concentration of theophylline in $B$. mori was similar to that in humans. The lethal dose and intestinal permeability can be considered to be different factors in drug characterization. Furthermore, the peaks that indicated the absorption of these compounds from the midgut could be detected by HPLC analysis approximately 30 min after oral administration.

Although theophylline and tetracycline might be substrates of some transporters, such as SLC22A6, SLC22A7, SLC22A8, SLC22A9 (25), it is unclear if these transporters are responsible for elimination of theophylline and tetracycline from the small intestine. $S L C 22 A 6$ is related to transport of tetracycline. $S L C 22 A 6$ is involved in the excretion of substrates in human. Additionally, SLC22A6 was highly expressed in B. mori, but, it was not expressed in the small intestine of the human. Thus, differences in the expression of drug transporters may cause differences in drug absorption. However, the $\mathrm{T}_{\max }$ of tetracycline in $B$. mori was consistent with that through the human small intestine. This result indicated that tetracycline could be evaluated by using a $B$. mori model without effects of SLC22A6. However, we only examined the expression of human homolog transporters in $B$. mori by using midgut RNA-seq data. In the future studies, it will be necessary to clarify where these transporters are expressed and how they are involved in drug absorption in the midgut cells in B.mori.

In addition, the absorption of these compounds might be affected by the peritrophic membrane in $B$. mori larvae. Pesticides with molecular weights of 50450 have shown oral toxicity in insects (26); thus, compounds with molecular weights of $\leq 500$ may be easily absorbed from the $B$. mori larval midgut. Using the isolated B. mori larval midgut, Hamamoto et al. (12) reported that the relationship between the polarity and molecular weight of a compound determines their intestinal permeability. In this experiment, we showed that membrane permeation in the midgut was induced by compounds that are more hydrophobic than hydrophilic and that membrane permeation was more likely induced by compounds of smaller molecular weight. Furthermore, Hamamoto et al. (12) mentioned that the isolated midgut of the $B$. mori model has similar intestinal permeability to those in humans and mice.

The human small intestine has a $\mathrm{pH}$ of 5-7 in the upper part and 7-8 in the lower part (27), whereas the $B$. mori larval midgut has a $\mathrm{pH}$ of approximately 11 (28). Differences in $\mathrm{pH}$ may cause structural changes in some compounds when it passes through the midgut. For this reason, when administering a compound, we should consider suitable solvents and adjust the $\mathrm{pH}$ inside the midgut to be equal to that of the human small intestine.

The method for evaluating the intestinal absorption of a compound used in this study is much simpler than the pre-existing intestinal perfusion system. Furthermore, the prediction of intestinal absorption of a compound via transporters would be more appropriate than existing methods because whole bodies were used in this study. As an advantage, B. mori can be maintained throughout the year by using an artificial diet, and large numbers of genetically homogeneous individuals can be kept in a narrow space at low cost. In addition, there are no animal ethics problems.

Prediction of the intestinal permeability of some compounds in humans is difficult because of species differences and bioavailability. If we can produce transgenic silkworms that express human drug transporters in the midgut, we will be able to solve this problem; therefore, B. mori larva is considered helpful for evaluation of the intestinal permeability of a compound at the screening stage. In sum, we propose the use of $B$. mori larvae to evaluate intestinal absorption of compounds. This system will be useful for evaluation of intestinal permeability in medical drug development.

\section{Acknowledgements}

The wild type silkworm strain o751 was obtained from the National Bio Resource Project KAIKO (NBRP KAIKO) at the Center of Genetic Resources at Kyushu University. Computations were partially performed on the NIG supercomputer at the ROIS National Institute of Genetics.

This work was supported by JSPS KAKENHI grant number JP26450465 to HT, grant number JP15H02483 to KiI and HT, and grant number JP15H02837 to RS and HT. This work was also supported by the NIG Collaborative Research Program (2014-A171 and 2015A155). This work was also supported by the National Bioscience Database Center (NBDC) of the Japan Science and Technology Agency (JST) to HB and TN.

\section{References}

1. Griffin B, O'Driscoll C. Models of the Small Intestine. In: In Situ Drug Absorption Studies: In Situ, In Vitro and In Silico Models: VII (Biotechnology: Pharmaceutical Aspects) (Ehrhardt C, Kim KJ, eds.). Springer, 2007; pp. 34-76.

2. Barthe L, Woodley J, Houin G. Gastrointestinal absorption of drugs: Methods and studies. Fundam Clin Pharmacol. 1999; 13:154-168.

3. Ishihara E, Usami M, Miura T, Tanigawa K. Glucose absorption from the small intestine in GK rats: A study of the isolated perfused intestine. J Jpn Diabetes Soc. 2001; 44:191-196.

4. Lennernäs H, Palm K, Fagerholm U, Artursson P. Comparison between active and passive drug transport in human intestinal epithelial (caco-2) cells in vitro and human jejunum in vivo. Int J Pharm. 1996; 127:103-107.

5. Mita K, Kasahara M, Sasaki S, et al. The genome sequence of silkworm, Bombyx mori. DNA Res. 2004; 
11:27-35.

6. Xia Q, Zhou Z, Lu C, et al. A draft sequence for the genome of the domesticated silkworm (Bombyx mori). Science. 2004; 306:1937-1940.

7. Suetsugu Y, Futahashi R, Kanamori H, et al. Large scale full-length cDNA sequencing reveals a unique genomic landscape in a lepidopteran model insect, Bombyx mori. G3 (Bethesda). 2013; 9:1481-1492.

8. Tomita M. Transgenic silkworms that weave recombinant proteins into silk cocoons. Biotechnol Lett. 2011; 33:645-654.

9. Wang F, Ma S, Xu H, Duan J, Wang Y, Ding H, Liu Y, Wang X, Zhao P, Xia Q. High-efficiency system for construction and evaluation of customized TALENs for silkworm genome editing. Mol Genet Genomics. 2013; 288:683-690.

10. Xia Q, Cheng D, Duan J, et al. Microarray-based gene expression profiles in multiple tissues of the domesticated silkworm, Bombyx mori. Genome Biol. 2007; 8:R162.

11. Kobayashi M. Fine structure of the basal granular cell in the midgut epitherium of the silkworm, Bombyx mori. J Seric Sci Jpn. 1971; 40:101-106.

12. Hamamoto H, Kurokawa K, Kaito C, et al. Quantitative evaluation of the therapeutic effects of antibiotics using silkworms infected with human pathogenic microorganisms. Antimicrob Agents Chemother. 2004; 48:774-779.

13. Hamamoto H, Kamura K, Razanajatovo IM, Murakami K, Santa T, Sekimizu K. Effects of molecular mass and hydrophobicity on transport rates through non-specific pathways of the silkworm larva midgut. Int J Antimicrob Agents. 2005; 26:38-42.

14. Hamamoto H, Tonoike A, Narushima K, Horie R, Sekimizu K. Silkworm as a model animal to evaluate drug candidate toxicity and metabolism. Comp Biochem Physiol C Toxicol Pharmacol. 2009; 149:334-339.

15. Kaito C, Akimitsu N, Watanabe H, Sekimizu K. Silkworm larvae as an animal model of bacterial infection pathogenic to humans. Microb Pathog. 2002; 32:183-190.

16. Kersey PJ, Allen JE, Armean I, et al. Ensembl genomes 2016 more genomes, more complexity. Nucleic Acids Res. 2015; 44:D574-D580.

17. Langmead B, Salzberg S. Fast gapped-read alignment with Bowtie 2. Nat Methods. 2012; 9:357-359.

18. Li B, Dewey CN. RSEM: Accurate transcript quantification from RNA-Seq data with or without a reference genome. BMC Bioinformatics. 2011; 12:323.

19. Ono H, Ogasawara O, Okubo K, Bono H. RefEx, a reference gene expression dataset as a web tool for the functional analysis of genes. Sci Data. 2017; 4:170105.

20. Finny DJ. Quantal response and the dosage response curve. In: Probit Analysis: A Statistical Treatment of the Sigmoid Response Curve. Cambridge University Press, Cambridge, UK, 1947; pp. 8-19.

21. Liu S, Zhou S, Tian L, Guo E, Luan Y, Zhang J, Li S. Genome-wide identification and characterization of ATPbinding cassette transporters in the silkworm, Bombyx mori. BMC Genomics. 2011; 12:491.

22. Tanaka S, Miyamoto K, Noda H, Jurat-Fuentes JL, Yoshizawa Y, Endo H, Sato R. The ATP-binding cassette transporter subfamily $\mathrm{C}$ member 2 in Bombyx mori larvae is a functional receptor for Cry toxins from Bacillus thuringiensis. FEBS J. 2013; 280:1782-1794.

23. Atsumi S, Miyamoto K, Yamamoto K, et al. Single amino acid mutation in an ATP-binding cassette transporter gene causes resistance to Bt toxin Cry1 Ab in the silkworm, Bombyx mori. Proc Natl Acad Sci U S A. 2012; 109:E1591- E1598.

24. Mukaiyama F, Ito T. Digestion experiments in the silkworm, Bombyx mori, by means of artificial diets. (I) Amount of food consumed, amount of food digested, and coefficient of digestibility. J Seric Sci Jpn. 1962; 31:317322.

25. Russel FGM. Chapter 2, Transporters: Importance in drug absorption, distribution, and removal. In: Enzymeand Transporter-Based Drug-Drug Interactions: Progress and Future Challenges (Pang, K. Sandy, Rodrigues, A. David, Peter, Raimund M. eds.), Springer, NY, U.S.A, 2010; pp. 27-49.

26. Ferrer I, Fernandez-Alba A, Zweigenbaum JA, Thurman EM. Exact-mass library for pesticides using a molecularfeature database. Rapid Commun Mass Spectrom. 2006; 20:3659-3668.

27. Soderind E, Dressman JB. Physiological factors affecting drug release and absorption in the gastrointestinal tract. In: Oral Drug Absorption: Prediction and Assessment (Dressman JB, Reppas C, eds.). CRC Press, NY, U.S.A, 2000; pp. 10-14.

28. Eguchi M, Kuriyama K, Daimon H. High alkalinity and function of proteases of digestive juice from the silkworm, Bombyx mori. J Seric Sci Jpn. 1986; 55:46-53.

(January 23, 2018; Revised February 10, 2018; Accepted February 21, 2018) 